\title{
Commentary on the benefits of alternative therapy: acupuncture as an effective intervention in treating cystic fibrosis
}

\section{Editorial}

Chinese Medicine is a systematic and comprehensive theoretical framework of a philosophical nature. Based on the recognition of the fundamental laws that govern the functioning of the human organism and its interaction with the environment according to nature's cycles. It seeks to apply this approachto the treatment of energy imbalances and the maintenance of health through various methods.

Based on Taoist concepts, for TCM, the individual is made up of a set of energies, coming from Heaven and Earth, that flow throughout the body and that must be in constant balance; when that doesn't happen, then we have the manifestation of pathologies, which we call energy imbalances.

Traditional Chinese Medicine (TCM), also known as Chinese Medicine in Chinese zhōngyaò xué, is usually given to conventional medical practices in China, developed throughout its history. TCM is used as alternative Medicine, with an integrative and complementary character, based on Taoist concepts. The theories that make up the $\mathrm{TCM}$ are Zang Fu, the five elements, and Yin and Yang. In contrast, the individual and the universe are inextricably linked. Based on the integration and interaction between human beings and nature. The state of health corresponds to the balance between the five elements and two other opposites (two opposing forces in constant YIN movement: governing the viscera and the YANG governing the organs). The imbalance corresponds to pathological manifestations or energy imbalances.

Cystic Fibrosis (CF) is a hereditary, lethal disease and more frequent in childhood and Caucasian populations, characterized mainly by infections and chronic obstruction of the respiratory and digestive system. The traditional physiotherapeutic approach has been an integral part of caring for this condition. One of the techniques used to improve breathing is cleaning the respiratory airway to remove excess bronchial secretions, the airways' resistance, and improved breathing in the short term.

According to Traditional Chinese Medicine, Cystic Fibrosis is due to the deficiency of pre-celestial Jing, existing in the essence of the Kidneys, which is acquired weakened by parents during conception, thus causing changes in the transformation and transport of Qi (energy), leading to patterns of disharmony. ${ }^{1}$

According to Kemp, ${ }^{2}$ MTC interprets Cystic Fibrosis in 2 phases, through vital energy: a) Qi - (Chi), or vital energy, the body requires this energy to function. Without Qi, the body dies. b) Yuan Qi - is a limited source with which we are born, used for life, and is stored in the kidneys. c) Postnatal Qi - the Qi that we continuously produce, made up of the air we absorb in the lungs and the food we eat through the stomach.

Due to the great suffering of patients caused by Cystic Fibrosis, the proposed theme is related to the intervention of Acupuncture in Patients with Cystic Fibrosis concerning the relief of pain and symptoms through anamnesis, identification of diagnosis, and
Volume 6 Issue I - 202I

\author{
Larry D Williams, Flavia Esther Avanci \\ Ph.D. Professor, NC Central University, USA \\ Social Worker Department of the Brazilian Military, Brazil \\ Correspondence: Larry D Williams, Ph.D. Professor, NC \\ Central University, USA, Tel (919) 672-4982 \\ Email Idwilliams@nccu.edu
}

Received: December 25, 2020 | Published: January 08, 2021

treatment. Acupuncture was the treatment indicated, as it provides a systemic and integral view of the human organism, which is influenced by circumstances and internal and external feelings. Treatment with acupuncture techniques aims at the systemic balance of the organism, better health conditions, and patient well-being. In this context, Acupuncture has to harmonize the Yīn and Yang murmurs' flow by stimulating points registered in the body. Acupuncture aims to restore the balanced functioning of the body and prevent pathologies and conditions. It is a method that stimulates specific points of the body with needles to restore or maintain health. ${ }^{3}$

Although not fully valued as an effective treatment, Acupuncture is widely applied in many health units and prescribed by many health professionals. Therefore, its treatment seeks to treat the compromised site in the body and act on the entire nervous system, stimulating the body's compensation and balance mechanism to heal the disease. In this context, from the stimulation of points, Acupuncture can change the dynamics of micro dilatations' regional circulation, providing muscle relaxation, healing spasm, decreasing inflammation and pain, release hormones such as cortisol, and as endorphins that promote analgesia. Besides, they increase resistance, normalize organic functions, and recover metabolism, which is very important in the healing body, soul, and spirit.

Traditional Chinese Medicine sees the individual as a whole, not just the symptoms of the disease presented. The treatment is individualized and specific. The diagnosis is made through physical, emotional, behavioral, and emotional observations taking into account the functioning of the organs, food, posture, stress, family, and social environment experienced by the patient and not only through the signs and symptoms as conventional Medicine proposes. Acupuncture allows for countless possibilities of interventions in different areas of the patient's life. Used both for the prevention of hereditary diseases and for the treatment of acute and chronic illnesses and pain. By having a more comprehensive and less invasive approach, the results are far reaching. Traditional Chinese Medicine goes beyond symptom relief, the purpose of treatment is to provide a cure by promoting a balance between body, mind and emotions, because the physical body is a reflection of emotions, beliefs, thoughts and feelings. Alternative Medicine through the diagnosis identifies deficiencies or emotional 
and behavioral excesses, changes in limiting patterns that cause imbalance, and triggers diseases that often conventional Medicine does not identify. Alternative Medicine understands the emotional causes associated with health problems and identifying what the disease is signaling, so that the appropriate treatment can be found to find a cure.

\section{Acknowledgments}

None.

\section{Conflicts of interest}

No potential conflict of interest relevant to this article was reported.

\section{References}

1. Claver, Steven. Physiology and pathology of organic fluids in traditional chinese medicine. Sao Paulo. Rocca, 2000.

2. Kemp JBS. Acupuncture and the treatment of cystic fibrosis. 2017.

3. Yamamura Y. Traditional acupuncture: the art of inserting. 2nd ed. São Paulo: Roca. 2001. 